\title{
Clinical Efficacy and Tolerability of Cimicoxib in Dogs with Osteoarthritis: A Multicentre Prospective Study
}

\author{
Joanna Murrell1 ${ }^{*}$, Erik Grandemange ${ }^{2}$, Frédérique Woehrle² ${ }^{2}$ Julie Menard ${ }^{3}$, Kate White ${ }^{4}$ \\ ${ }^{1}$ School of Veterinary Sciences, University of Bristol, Bristol, UK \\ ${ }^{2}$ Vétoquinol S.A., Centre de Recherche, Lure, France \\ ${ }^{3}$ Vétoquinol S.A., Paris, France \\ ${ }^{4}$ School of Veterinary Medicine and Science, University of Nottingham, Nottingham, UK \\ Email: ${ }^{*}$ jo.murrell@bristol.ac.uk
}

Received 10 March 2014; revised 10 April 2014; accepted 25 April 2014

Copyright (C) 2014 by authors and Scientific Research Publishing Inc.

This work is licensed under the Creative Commons Attribution International License (CC BY).

http://creativecommons.org/licenses/by/4.0/

(c) (i) Open Access

\section{Abstract}

Background: Cimicoxib is a coxib recently licensed in Europe for pain and inflammation associated with osteoarthritis $(\mathrm{OA})$, and the management of perioperative pain due to orthopaedic or soft tissue surgery. Purpose: This prospective study was to complete the product information for the end users by providing additional scientific data obtained after a thirty-day treatment course of cimicoxib in dogs with $\mathrm{OA}$, and to collect owners' feedback. Data were collected from nine European countries with 492 client owned dogs recruited to the trial. Dogs were treated once daily with $2 \mathrm{mg} / \mathrm{kg}$ cimicoxib orally. Immediately before, at Day (D) 15 and D30 after the start of treatment veterinarians and owners scored body condition, appetite, locomotion, lameness, pain on palpation and manipulation of the joint and joint effusion (veterinarians) and dog demeanor and well being (owners). In a subset of dogs, serum urea $(n=191)$, creatinine $(n=184)$, AST $(n=141)$ and ALT ( $n=174)$ were measured at day (D) 0 and D30. Statistical tests were carried out to detect significant changes in the clinical parameters with time. Results and Discussion: Veterinary and owner assessments were analysed from 236 and 215 dogs respectively. Improvements in locomotion, mobility, pain scores and dog demeanor and body condition were identified; outcome measures assessed by veterinarians continued to improve after 15 days of treatment up to the 30-day time point. At D30 a significantly higher number of dogs had an urea concentration superior to the upper limit of the reference range. However, there was no significant difference for creatinine, ALT and AST. Conclusions: A 30-day treatment course with cimicoxib improved locomotion and decreased pain scores in dogs with $\mathrm{OA}$, with minimal adverse effects. These data, support pre-clinical data in dogs receiving cimicoxib and are useful for veterinarians making decisions about which NSAID to administer to dogs that require pain management for $\mathrm{OA}$.

\footnotetext{
*Corresponding author.
} 


\section{Keywords}

Osteoarthritis, Dogs, NSAID, Cimicoxib, Pain, Analgesia, Owner, Kidney, Renal, Mobility

\section{Introduction}

Osteoarthritis (OA) is the most commonly reported non traumatic orthopaedic condition of dogs in the United Kingdom; studies indicate that 1 in 5 dogs over one year of age suffer from this painful and debilitating disease [1] and this prevalence increases with age and additional factors such as obesity [2]. Several drug classes are available for management of OA, including corticosteroids, Non Steroidal Anti-Inflammatory Drugs (NSAIDs), disease modifying agents and neutraceuticals.

NSAIDs are routinely used for long-term pain relief in dogs with OA, and there are a variety of different NSAID molecules available with market authorization for administration to dogs. NSAIDs can be classified according to their ratio of selectivity for the COX-1 and COX-2 enzymes [3], with a drive towards generation of COX-2 selective NSAIDs, termed coxibs [3]. The three most recently licensed NSAIDs for dogs; firocoxib, robenacoxib and cimicoxib are all classed as coxibs, although the clinical benefits of choosing coxibs over other less selective NSAIDs in dogs are not yet quantified. Control of the pain associated with OA is best achieved using a multimodal approach combining pharmaceutical and supportive therapy to slow the progression of the disease [4].

There is a large structural diversity in the NSAIDs available for prescribing to dogs, but a lack of comprehensive studies comparing the different formulations hinders decision making about which NSAID to prescribe to a particular dog. Gastrointestinal side effects such as vomiting and diarrhoea may be less frequent with the newer, more COX-2 selective NSAIDs, but a paucity of large clinical studies comparing all the different NSAIDs makes these statements difficult to prove beyond doubt [3]. Furthermore, comparing the analgesic efficacy of different NSAIDs is limited by the subjective assessment of pain and wellbeing. Currently, there is no standard method for assessing OA in dogs and discrepancies between owners and veterinarians assessments are well documented [5].

Cimicoxib is a coxib recently licensed in Europe for the long-term management of pain and inflammation associated with $\mathrm{OA}$, and the management of perioperative pain due to orthopaedic or soft tissue surgery. The tablet can be administered prior to surgery and continued in the postoperative period. In clinical trials, cimicoxib was comparable to carprofen for post-surgical pain, and to firocoxib for pain associated with chronic OA [6]. The purpose of this prospective study was to complete the product information for the end users by providing additional scientific data obtained after a thirty-day treatment course of cimicoxib in dogs with OA, and to collect owners' feedback.

\section{Materials and Methods}

This was a prospective clinical study in which all dogs received the test drug cimicoxib; veterinarians and owners were therefore not blinded to treatment administration. The study was conducted in nine European countries with four hundred and ninety two client owned dogs with clinical signs of OA recruited to the trial (see Table 1 for inclusion and exclusion criteria).

Table 1. Inclusion and exclusion criteria that determined whether a dog could be recruited to the study.

\begin{tabular}{|c|c|c|}
\hline & Inclusion criteria & Exclusion criteria \\
\hline \multirow{4}{*}{ Criteria } & Dogs $>10$ weeks age & $\begin{array}{l}\text { Dogs suffering from gastrointestinal } \\
\text { disorders or haemorrhagic disorders }\end{array}$ \\
\hline & Dogs weighing a minimum of $3 \mathrm{~kg}$ & $\begin{array}{c}\text { Dogs treated with corticosteroids or other } \\
\text { NSAIDs }\end{array}$ \\
\hline & $\begin{array}{l}\text { Dogs presenting with lameness associated with OA lesions and } \\
\text { progressing for more than one month }\end{array}$ & Pregnant and lactating bitches \\
\hline & $\begin{array}{l}\text { Xray confirmation of OA lesions } \\
\text { (if considered necessary by the clinician) }\end{array}$ & \\
\hline
\end{tabular}


Veterinarians working in 106 private first opinion veterinary practices were approached to participate in study, each veterinarian was requested to collect data from at least three dogs treated with cimicoxib with a diagnosis of OA, with a maximum of six dogs recruited per veterinarian. For the majority of veterinary practices only one veterinarian per practice was involved in the study. A diagnosis of OA was based on clinical examination; signs of pain and lameness must have been present for more than a month. The veterinarian directly approached the dog owner during a veterinary consultation about whether the owner was willing to allow their dog to be recruited to the trial if their dog met the inclusion criteria. The owners also completed a questionnaire during the trial and were therefore fully informed about the trial and study conditions; written consent from the owner was obtained prior to recruitment. Prescription diets that had commenced over a month prior to the trial starting were tolerated but no diet changes within the trial period were permitted.

All dogs were treated with cimicoxib, $2 \mathrm{mg} / \mathrm{kg}$ once daily per os for thirty days, with dogs evaluated on day (D) D0, immediately before the start of treatment, D15 and D30 by the veterinarian and the dog owner. All study information was provided in the native language for each European country. With the exception of serum biochemistry, all assessments described below were carried out at each time point.

\subsection{Veterinarian Assessment}

General condition, appetite, locomotion, lameness, pain on palpation and manipulation of the joint and palpable joint effusion were scored using a 4 point ordinal and simple descriptive scale (Table 2).

Table 2. Clinical scoring criteria used to score different outcome measures in dogs before and at 15 and 30 days after the start of treatment with cimicoxib by veterinarians.

\begin{tabular}{|c|c|c|}
\hline \multicolumn{3}{|c|}{ Clinical scoring criteria } \\
\hline General condition & Normal & 0 \\
\hline & Slightly altered & 1 \\
\hline & Severely altered & 2 \\
\hline & Prostation/apathy & 3 \\
\hline \multirow[t]{4}{*}{ Appetite } & Normal & 0 \\
\hline & Slightly decreased $(<50 \%)$ & 1 \\
\hline & Severely decreased $(\geq 50 \%)$ & 2 \\
\hline & Anorexia & 3 \\
\hline \multirow[t]{4}{*}{ Locomotion $^{*}$} & Normal & 0 \\
\hline & Slightly decreased (<25\%) & 1 \\
\hline & Moderately decreased (25\% - 50\%) & 2 \\
\hline & Severely decreased (>50\%) & 3 \\
\hline \multirow[t]{4}{*}{ Lameness } & Absent & 0 \\
\hline & Slight: intermittent abnormal walk & 1 \\
\hline & Moderate: continuous abnormal walk and/or intermittent no weight bearing & 2 \\
\hline & Severe: no weight bearing & 3 \\
\hline \multirow[t]{4}{*}{$\begin{array}{l}\text { Pain at palpation and } \\
\text { manipulation of the joint }\end{array}$} & Absent & 0 \\
\hline & Slight: induced only when fully extending and/or flexing the joint & 1 \\
\hline & Moderate: induced by minimum palpation & 2 \\
\hline & Severe: manipulation impossible or very strong reaction at joint palpation & 3 \\
\hline \multirow[t]{4}{*}{ Joint effusion } & Absent & 1 \\
\hline & Slight: induced only when fully extending and/or flexing the joint & 2 \\
\hline & Moderate: Obvious joint effusion fluctuating at palpation & 3 \\
\hline & Severe: pronounced joint effusion fluctuating at palpation & 4 \\
\hline
\end{tabular}

${ }^{*}$ Quantitative point of view, amount of movement(s), average distance covered. To be assessed according to the owner's history, and compared to the previous health status of the dog. 
Veterinarians in each country were trained by a single study coordinator about how to carry out the assessments in a standardized manner, written instructions were also provided to the veterinarian describing the study protocol. In this study, the term "general condition" related to demeanor and mentation, rather than a change in body weight (i.e. change in body condition score), weight was not monitored throughout the study period, although over 30 days significant changes in bodyweight in otherwise healthy dogs would not be expected to occur. Although not a prerequisite for inclusion in the study, blood samples could be collected for measurement of serum urea, creatinine, aspartate transaminase (AST) and alanine aminotransferase (ALT) at D0 and D30, any samples collected were analysed at the laboratory normally used by the veterinary practice. Incidence of side effects in treated dogs were recorded by the veterinarians on their forms, based on the discussion with the pet owner, at D15 and D30.

\subsection{Owner Assessment}

Owners were asked to complete a diary (Table 3) about changes in their dog's demeanor and well-being at the same time points as the veterinary assessments. Similarly to the veterinary assessments, an ordinal and descriptive scale was used to rate physical condition, appetite, pain behaviours and walking ability, in addition to a Numerical Rating Scale to quantify pain and overall satisfaction with cimicoxib. Further data assessing the occurrence of vomiting and/or diarrhoea, ease of drug administration and overall satisfaction with the treatment regime were also collated.

\subsection{Statistical Analysis}

Data are described in tables with means and standard deviations for quantitative variables, and frequencies/percentages for qualitative variables. Statistical tests were carried out to detect significant changes in the clinical parameters within time. A global Chi-square test from D0 to D30 was performed. If the test was significant at the 5\% level, then $2 \times 2$ comparisons using chi-square tests were implemented: D0 versus D15, D0 versus D30, D15 versus D30. For $2 \times 2$ comparisons, the significance level was set at $1.67 \%$ (i.e. $5 \% / 3$ ) in order to protect the $5 \%$ global first type error. For tolerability parameters, frequencies prior and at the end of treatment were compared with Fisher's exact test at the 5\% significance level. Biochemical data are presented as frequencies, but were not subjected to statistical analysis. No sample size calculation was implemented prior to starting the study. Commercial software packages were used for the analysis of the evaluation of the clinical scores ${ }^{1}$ and for the analysis of the tolerability and biochemical data ${ }^{2}$.

Table 3. Clinical scoring criteria used to score different outcome measures in dogs before and at 15 and 30 days after the start of treatment with cimicoxib by pet owners.

\begin{tabular}{|c|c|c|}
\hline \multicolumn{3}{|c|}{ Clinical scoring criteria } \\
\hline How would you rate your dog's condition? & Normal, as usual & 0 \\
\hline & Abnormal & 1 \\
\hline \multirow[t]{3}{*}{ How would you rate your dog’s appetite? } & Normal & 0 \\
\hline & Decreased & 1 \\
\hline & No appetite & 2 \\
\hline \multirow[t]{3}{*}{$\begin{array}{l}\text { Which of the following statements best describes } \\
\text { your dog's behavior? }\end{array}$} & He/she is a happy animal that plays, reacts with enthusiasm when called & 0 \\
\hline & He/she is an anxious animal, less responsive when called & 1 \\
\hline & He/she does not respond when called & 2 \\
\hline \multirow[t]{3}{*}{ Does your dog have difficulties walking? } & Not at all & 0 \\
\hline & $\mathrm{He} /$ she has difficulties but can still support some weight on one leg & 1 \\
\hline & He/she has difficulties and he/she cannot put any weight on the leg & 2 \\
\hline \multirow[t]{3}{*}{ Does your dog express any pain by crying? } & Not at all & 0 \\
\hline & Yes but/she stops crying when being comforted by being spoken to & 1 \\
\hline & Yes and he/she does not stop despite being comforted by being spoken to & 2 \\
\hline
\end{tabular}

${ }^{1}$ SYSTAT, version 10, SPSS Inc., Chicago, Ill; ${ }^{2}$ SAS, version 9.2, SAS Institute Inc., Cary, NC. 


\section{Results}

Four hundred and ninety two dogs were enrolled on the study, but veterinary and owner assessment data were analysed from 236 (48\%) and 215 (46\%) cases only due to failure to collect data from at least one of the two assessment time points (15 and 30 days) following the start of treatment, by either the veterinarian or owner. Incomplete demographic data were collected from some dogs, the number of dogs for which data were available is indicated in brackets where appropriate.

Dogs were recruited in nine European countries, with most dogs recruited in Germany $(n=60)$, UK $(n=44)$ and Italy $(\mathrm{n}=42)$. The mean (minimum-maximum) age and bodyweight of the dogs were $8.9(0.6$ - 18.0) years $(n=206)$ and $26.6(5.0-70.0) \mathrm{kg}(\mathrm{n}=209)$ respectively. Seventy five dogs were intact males $(35 \%), 38$ dogs were intact females (18\%), 48 (23\%) and 50 (24\%) dogs were neutered male and female dogs respectively (n = 211). There was a wide distribution of breeds, with 37 different breeds represented by on average one to three dogs each. The exception was cross breed dogs, of which there were 28. The distribution of joints affected by OA in recruited animals is shown in Table 4.

\subsection{Veterinary OA Questionnaire}

The proportion of dogs assigned to each category (i.e. 0 to 3 or 1 - 4) for each of the clinical criteria was compared at the three assessment time points (D0, D15, D30). General condition improved with time, with $60.5 \%$ of dogs judged to have normal condition at the first assessment (D0), and 80.0\% and 85.1\% of dogs judged to have normal condition at D15 and D30 respectively (Figure 1). The proportion of dogs assigned to each general condition score changed significantly between D0 and D15, and D0 and D30 ( $<<0.001$ ), but not between D15 and

Table 4. Distribution of joints affected by OA in recruited dogs. Assessment was based on clinical examination with or without additional radiographic examination.

\begin{tabular}{cc}
\hline \multicolumn{1}{c}{ Clinical examination } & Number of dogs \\
\hline Joints affected with OA (unilateral or bilateral disease) & 32 \\
Stifle (s) and Elbow (s) & 37 \\
Hip (s) and Spinal column & 26 \\
Elbow (s) and Hip (s) & 67 \\
Stifle joint (s) with at least one other joint affected & 44 \\
Spinal column & 206 \\
Total number of dogs &
\end{tabular}

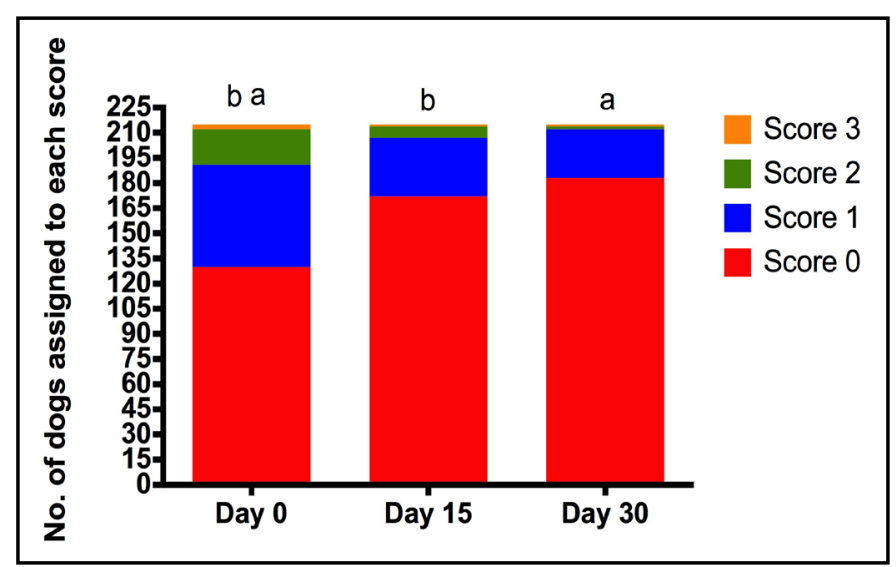

Figure 1. Number of dogs assigned to each general condition score (0 normal to 3 (prostration/apathy) at each of the assessment time points (Day 0 (D0), Day 15 (D15), Day 30 (D30)) by veterinarians. Total number of dogs assessed was 215 . The letters indicate a statistically significant difference between the proportion of dogs in each of the categories for condition score between the different time points $(\mathrm{a}=\mathrm{p}<0.001$ and $\mathrm{b}=\mathrm{p}<0.001)$. 
D30 ( $=0.204)$. When the population of dogs was considered, locomotion scores significantly improved at both D15, relative to D0; and at D30, relative to D15 with the percentage of dogs assigned a score of normal locomotion increasing from $2.3 \%$ at D0 to $44.2 \%$ at D30 ( $<$ 0.001) (Figure 2). Lameness scores decreased over the treatment period, with the percentage of dogs with no lameness increasing from $4.2 \%$ at D0 to $26.5 \%$ at D15 and $43.7 \%$ on D30 ( $<0.0001$ ) (Figure 3). In agreement with the lameness scores, pain associated with joint manipulation decreased between D0 and D15 and between D15 and D30 ( $<0.001$ and p $<0.006$ respectively) (Table 5). Palpable effusion of the joint decreased significantly between D0 and D15 but further improvement between D15 and D30 was not significant (Table 5).

There was no significant change in the appetite of dogs during the course of treatment (data not shown), with the majority of dogs judged to have a normal appetite at all assessment time points.

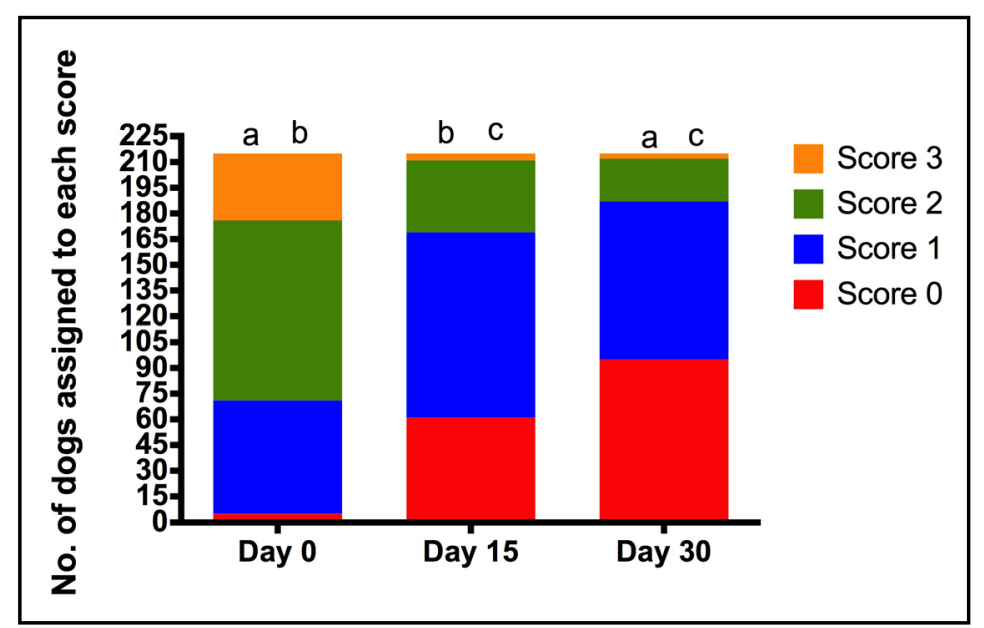

Figure 2. Number of dogs assigned to each locomotion score (0 normal to 3 (severely decreased) at each of the assessment time points (Day 0 (D0), Day 15 (D15), Day 30 (D30)) by veterinarians. Total number of dogs assessed was 215 . The letters indicate a statistically significant difference between the number of dogs assigned each score at each of the assessment time points $(\mathrm{a}=\mathrm{p}<0.001, \mathrm{~b}=\mathrm{p}<0.001, \mathrm{c}=\mathrm{p}=0.001)$.

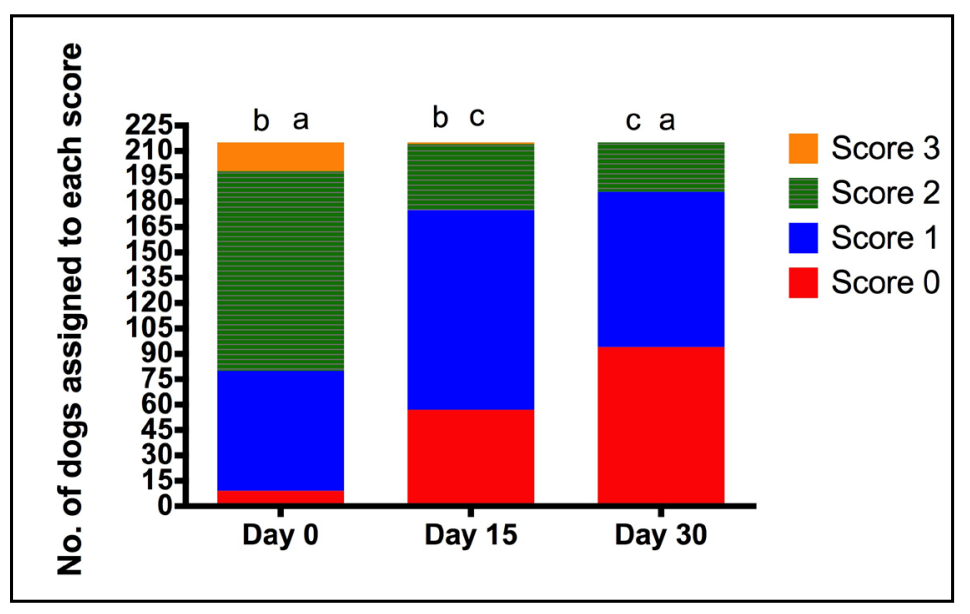

Figure 3. Number of dogs assigned to each lameness score (0 normal to 3 (severe lameness, no weight bearing) at each of the assessment time points (Day 0 (D0), Day 15 (D15), Day 30 (D30)) by veterinarians. Total number of dogs assessed was 215. The letters indicates a statistically significant difference between the number of dogs assigned to each score at the different assessment time points $(\mathrm{a}=\mathrm{p}<0.0001, \mathrm{~b}=\mathrm{p}<0.001, \mathrm{c}=\mathrm{p}=$ 0.001). 
Table 5. Number (\%) of dogs assigned to each score for pain on joint manipulation (0 no pain on joint manipulation to 3 (severe pain, manipulation impossible or very strong reaction to joint palpation) and joint effusion (0 no joint effusion to 3 severe joint effusion that is pronounced and fluctuates at joint manipulation) at each of the assessment time points (Day 0 (D0), Day 15 (D15), Day 30 (D30)) by veterinarians. Total number of dogs assessed was 215 . The paired symbols ${ }^{*}$ and ${ }^{\wedge}$ indicate a statistically significant difference between assessment time points for the number of dogs assigned to each category score $\left({ }^{*} \mathrm{p}<0.001 .{ }^{\wedge} \mathrm{p}=0.006,{ }^{\mathrm{a}} \mathrm{p}<0.001,{ }^{\mathrm{b}} \mathrm{p}<0.001\right)$.

\begin{tabular}{|c|c|c|c|c|c|c|}
\hline & D0 & D15 & D30 & D0 & D15 & D30 \\
\hline & $\begin{array}{l}\text { Pain on joint } \\
\text { manipulation }\end{array}$ & $\begin{array}{l}\text { Pain on joint } \\
\text { manipulation }\end{array}$ & $\begin{array}{l}\text { Pain on joint } \\
\text { manipulation }\end{array}$ & Joint effusion & Joint effusion & Joint effusion \\
\hline $\begin{array}{l}\text { Number (\%) of dogs } \\
\text { with score } 0\end{array}$ & $17(7.91)$ & 73 (33.9) & $103(47.9)$ & $133(61.7)$ & $178(82.8)$ & $187(87.0)$ \\
\hline $\begin{array}{l}\text { Number (\%) of dogs } \\
\text { with score } 1\end{array}$ & $74(34.2)$ & $113(52.6)$ & $98(45.6)$ & $54(25.1)$ & 30 (13.9) & $26(12.1)$ \\
\hline $\begin{array}{l}\text { Number (\%) of dogs } \\
\text { with score } 2\end{array}$ & $97(41.1)$ & 27 (12.6) & $14(6.5)$ & $24(11.2)$ & $7(3.3)$ & $2(0.9)$ \\
\hline $\begin{array}{c}\text { Number (\%) of dogs } \\
\text { with score } 3\end{array}$ & 27 (12.6) & $2(0.9)$ & $0(0.0)$ & $4(1.9)$ & $0(0.0)$ & $0(0.0)$ \\
\hline Total number of dogs & $215^{* \wedge}$ & $215^{\wedge}$ & $215^{*}$ & $215^{\mathrm{ab}}$ & $215^{\mathrm{b}}$ & $215^{\mathrm{a}}$ \\
\hline
\end{tabular}

\subsection{Biochemical Results}

Serum urea, creatinine, AST and ALT were measured in a subset of dogs in the study on D0 and D30. The number of dogs with a serum concentration below a defined physiological limit (normal) at the D0 and D30 are presented in Table 6 .

\subsection{Owner Assessment}

Pain score decreased significantly over the course of treatment; median (minimum-maximum) pain scores ( 0 , no pain-10, unbearable pain) at the start of treatment (D0) and D30 were $6.0(6.0-10)$ and $2.0(2.0$ - 8.0) respectively ( $<<0.0001)$. This is in agreement with the change in walking ability and expression of pain by crying between D0 and D30. The percentage of dogs that had no difficulty walking increased from $6.4 \%$ (D0) to $54.7 \%$ (D30) ( $<$ 0.0001), while the percentage of dogs that expressed pain by crying and did not stop crying when spoken to or comforted decreased from 7.2\% (D0) to 0\% (D30) (p < 0.001) (Figure 4 \& Figure 5). The behaviour of the dog could be rated from happy and enthusiastic to non-responsive when called. The percentage of dogs that were happy and enthusiastic increased from $47.9 \%$ to $84.8 \%$ on days 0 and 30 respectively, whilst the percentage of dogs that were non-responsive decreased from $8.1 \%$ to $2.1 \%$ over the same time course ( $\mathrm{p}<$ 0.0001) (Figure 6). In contrast to the veterinary assessment of appetite over the 30 day treatment period, owners reported an increase in the percentage of dogs with a normal appetite (as opposed to a decrease or no appetite) (78.8\% D0, 91.5\% D30) (p < 0.0001).

At the end of the study period (D30) owners expressed a high level of satisfaction about the treatment of their dog with cimicoxib. Seventy seven percent of owners either agreed or totally agreed with the statement that cimicoxib treatment noticeably reduced their dog's signs of pain, and $85.6 \%$ of owners either agreed or totally agreed with the statement that their dog had no visible side effects (such as vomiting or diarrhoea) during the study. Seventy three percent of owners either agreed or totally agreed with the statement that their dog ate cimicoxib spontaneously.

\section{Discussion}

The study aim was to complete the scientific information available for cimicoxib in the registration file [6] [7] with additional scientific data on clinical efficacy from daily use in routine practice in Europe, in a population of dogs with OA following thirty days of treatment. Efficacy and safety were demonstrated during registration studies and this prospective clinical trial was closer to a survey than to a field clinical trial, it was not the intention to carry out a blinded, positively controlled (using a comparator licensed NSAID) trial in general practice. Field clinical trials performed during the registration process are strictly standardized and have to respect specific guidelines (e.g. Good Clinical Practice, biostatistical guidelines). Some limitations of such studies are that 
Table 6. Number (\%) of dogs with an elevated and normal serum concentration of urea, creatinine, ALT and AST at D0 and D30. The total number of dogs for which data were available for each parameter are indicated.

\begin{tabular}{|c|c|c|c|c|c|c|c|c|}
\hline \multirow[b]{2}{*}{ D0 } & \multicolumn{2}{|c|}{$\begin{array}{c}\text { Urea }(\mathrm{mmol} / \mathrm{L}) \\
\mathrm{N}=191\end{array}$} & \multicolumn{2}{|c|}{$\begin{array}{c}\text { Creatinine }(\mu \mathrm{mol} / \mathrm{L}) \\
\mathrm{N}=184\end{array}$} & \multicolumn{2}{|c|}{$\begin{array}{l}\mathrm{ALT}(\mathrm{IU} / \mathrm{L}) \\
\mathrm{N}=174\end{array}$} & \multicolumn{2}{|c|}{$\begin{array}{l}\mathrm{AST}(\mathrm{IU} / \mathrm{L}) \\
\mathrm{N}=141\end{array}$} \\
\hline & $<9.1$ & 166 (87) & $<138.4$ & 169 (92) & $<57.3$ & $123(71)$ & $<48.5$ & $110(78)$ \\
\hline D0 & $\geq 9.1$ & $25(13)$ & $\geq 138.4$ & 163 (88.6) & $\geq 57.3$ & 51 (29) & $\geq 48.5$ & $31(22)$ \\
\hline D30 & $<9.1$ & 147 (77) & $<138.4$ & $15(8)$ & $<57.3$ & $122(70)$ & $<48.5$ & $113(80)$ \\
\hline D30 & $\geq 9.1$ & $44(23)$ & $\geq 138.4$ & $21(11.4)$ & $\geq 57.3$ & $52(30)$ & $\geq 48.5$ & $28(20)$ \\
\hline $\mathrm{p}$ value & \multicolumn{2}{|c|}{$\mathrm{p}=0.0162$} & \multicolumn{2}{|c|}{$p=0.38$} & \multicolumn{2}{|c|}{$\mathrm{p}=1.00$} & \multicolumn{2}{|c|}{$\mathrm{p}=0.77$} \\
\hline
\end{tabular}

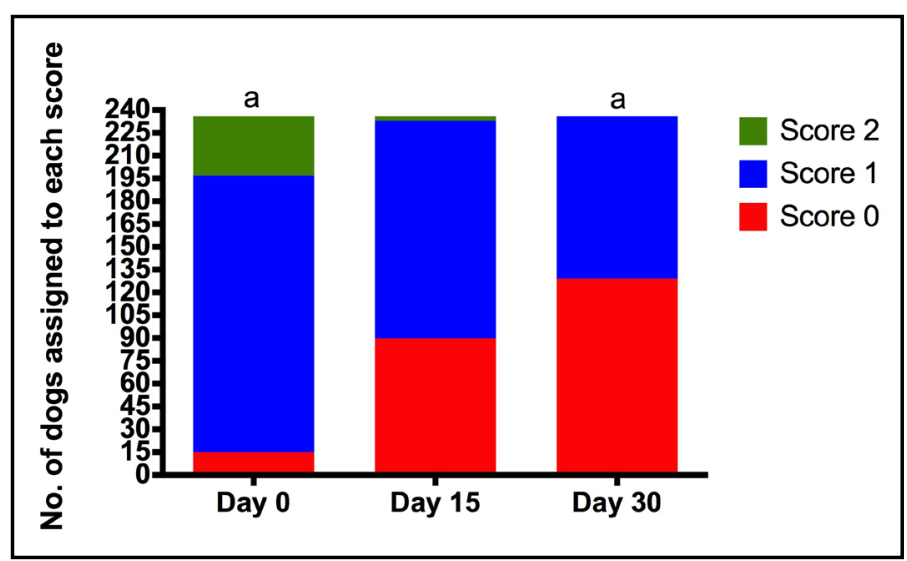

Figure 4. Number of dogs assigned to each category for assessment of difficulty walking ( 0 normal to 2 (he/she has difficulties walking and cannot put any weight on the leg) at each of the assessment time points (Day 0 (D0), Day 15 (D15), Day 30 (D30)) by owners. Total number of dogs assessed was 236. a indicates a statistically significant difference between the number of dogs assigned each score at each of the assessment time points $(\mathrm{p}<0.0001)$.

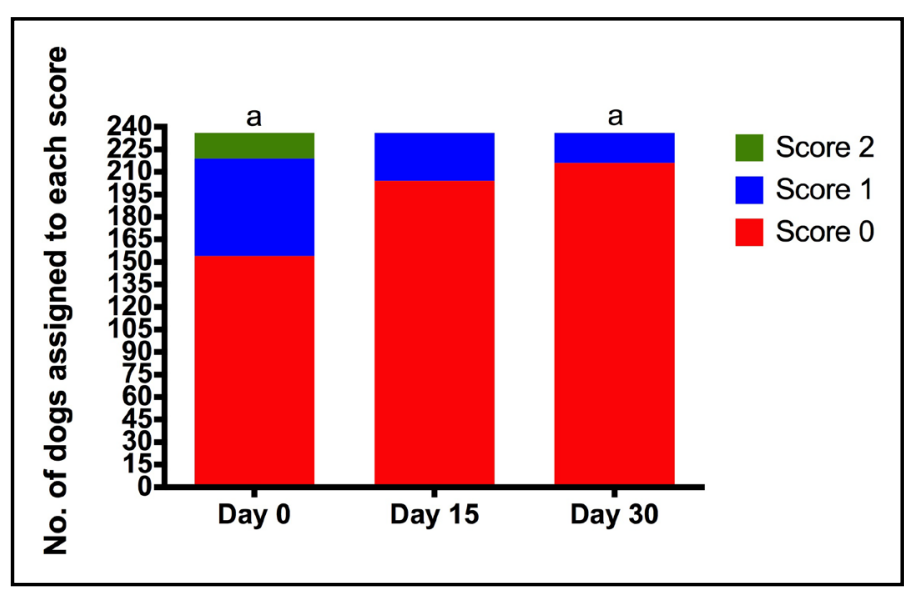

Figure 5. Number of dogs assigned to each category for assessment of expression of pain by crying ( 0 no expression of pain by crying) to 2 (expression of pain by crying that does not stop despite being comforted by being spoken to) at each of the assessment time points (Day 0 (D0), Day 15 (D15), Day 30 (D30)) by owners. Total number of dogs assessed was 236. a indicates a statistically significant difference between the number of dogs assigned each score at each of the assessment time points $(\mathrm{p}<0.0001)$. 


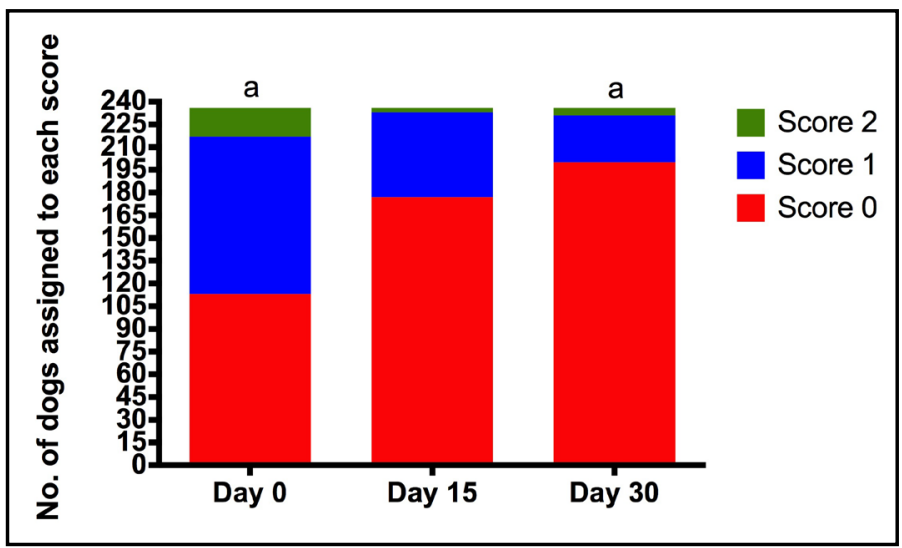

Figure 6. Number of dogs assigned to each category for behaviour ( 0 a happy animal that plays and reacts with enthusiasm when called) to 2 (an animal that does not respond when called) at each of the assessment time points (Day 0 (D0), Day 15 (D15), Day 30 (D30)) by owners. Total number of dogs assessed was 236. a indicates a statistically significant difference between the number of dogs assigned each score at each of the assessment time points $(\mathrm{p}<0.0001)$.

sometimes they are not in line with the current field practice and the sample size can be limited [8]. In the current field survey both veterinarians and dog owners were asked to complete a questionnaire before, after fifteen and thirty days of therapy in order to try and obtain a broad spectrum of data relating to both change in pain intensity following the onset of treatment and change in dog well-being. A statistically significant clinical improvement, as judged by the veterinarian and owner was found over the thirty-day period. The survey also provides additional tolerability data in a large population of dogs suffering from OA. This approach increases the inter-observer variability but does allow inclusion of a larger veterinarian population which may be more representative of the current practice in treating osteoarthritic pain in dogs across Europe.

The intention of the study was to recruit a large number of dogs with OA across a number of European countries. In order to reflect current clinical $1^{\text {st }}$ opinion veterinary practice, a diagnosis of OA was based on clinical assessment of the patient and the presence of pain on joint manipulation. In addition the joints affected with OA were not restricted for inclusion in this study, reflecting the target population of dogs that would likely be treated with cimicoxib in the field. Although dogs older than 10 weeks of age were eligible for recruitment, the median age of the study cohort indicates that older dogs were most commonly recruited to the study, supporting a diagnosis of OA as the underlying cause of joint pain. The adoption of a multicentre approach has been proposed as a way of securing transparent quality control in data collection [9], however each veterinarian that participated in the study was requested to recruit only three cases such that the number of different people carrying out clinical pain assessments was large. Training in carrying out the assessments was provided to the veterinarians prior to the start of the study (but not the pet owners) in the use of the scoring systems; any effect of inter-observer variability will have been somewhat ameliorated by the repeated measures study design, whereby all dogs were compared over time by the same veterinarian and per owner to measurements collected on D0.

The type of data collected from veterinarians was based on a Simple Descriptive Scale (SDS) relating to general aspects of dog well-being (e.g. appetite and general condition) as well as more specific aspects relating to lameness and pain caused by OA (e.g. locomotion and lameness scores, response to joint manipulation and palpable effusion of the joint). The SDS comprised four possible descriptors for each category that was then assigned a score from 0 - 3. Similar scoring systems have been used in other studies to investigate analgesic efficacy of NSAIDs in dogs [10] [11]. Simple descriptive scales are simple and quick to use, which makes them attractive for field type studies where data are collected as part of a veterinary consultation, but lack sensitivity compared with Numerical Rating and Visual Analogue Scales. Numerical Rating Scales have been recommended to quantify acute pain in dogs because this type of scoring system is more sensitive than a SDS, but less vulnerable to inter-observer variability than Visual Analogue Scales [12]. The optimal scoring system for assessment of chronic pain has not been systematically evaluated in veterinary studies in dogs, and will likely depend on the nature of the outcome measure and the study design. It is important that any scoring system used to 
quantify pain is both reliable and valid [13] and neither reliability nor validity of the owner and veterinarian questionnaires was assessed before the start of data collection. However, given the similarity between the scoring criteria used in the present investigation to those incorporated into other studies it is not unreasonable to assume that the questions asked of the owners and veterinarians related to chronic pain in the dog. For example, questions relating to mood, willingness to play, appetite, audible complaining, lameness, stiffness, and pain, were incorporated in the questionnaires used by veterinarians and owners in the present study, all of which are included in questionnaires that have been previously used in the objective assessment of pain associated with OA [14]-[17].

A strength of the study was that a group of dogs with OA was recruited with no bias towards selecting animals of a particular breed, sex or age, thereby likely reflecting the field population of dogs with OA in Europe. Improvements in general mobility and joint movement and effusion were found by veterinarians, likely as a result of the anti-inflammatory and anti-hyperalgesic effects of NSAID molecules [18]. Collection of data over a thirty day time period allowed the change in each outcome measure between D0 and D15 and D15 and D30 to be compared, in order to ascertain the time period over which continued improvement in a particular outcome variable can be expected to occur following the start of NSAID treatment. In this population of dogs, joint effusion did not improve further after the first 15 days of treatment whereas the outcome measures of locomotion and lameness scores as well as pain on joint manipulation improved between D0 and D15 and between D15 and D30. This may relate to a differential time course of peripheral and central antihyperalgesic and anti-inflammatory effects of NSAIDs [19]. There is a paucity of data on the time course of analgesia following NSAID treatment in dogs [20], but these data suggest that prescribing cimicoxib for longer than 15 days is necessary in order to obtain the maximum treatment benefit from the drug. The benefits of continuing treatment for longer than 30 days in terms of continuing improvement in outcome measures were not evaluated in the present study. The use of coxibs for long term treatment of OA in humans has been investigated [21] but there are very limited data for long term use of coxibs in dogs [8].

The importance of owner assessments of chronic pain in dogs are being increasingly emphasized in veterinary studies [13] [22] and a number of different questionnaires have been developed specifically to measure pain associated with OA in dogs, such as the Helsinki Chronic Pain Index [14] [23] and Canine Brief Pain Inventory [15]. However differences between owner and veterinarian assessments have been reported in dogs treated surgically for cranial cruciate ligament deficiency [24]. The present study used different questionnaires for the owners and veterinarians, with the owner questionnaires placing greater emphasis on questions that might provide information about dog well being rather than lameness per se. Owners reported their dogs to show improvements in demeanor and walking before and after 30 days of treatment, although the contribution of a placebo effect is unknown, thus mirroring the improvements reported by veterinarians over a similar time period. A confounding factor was the relative timing of the veterinary and owner assessments.

The data collection and the coding system did not allow determination of the degree of matching between the owner and veterinary questionnaires and whether or not they were related to the same dogs. Therefore it is possible, although unlikely, that a different population of dogs is described by the owner and the veterinarian. There were no reports of dogs being withdrawn from the study due to NSAID related side effects, although the precise causes of the missing data are unknown. The decision to withdraw a case from the analysis pool was a result of incomplete questionnaires either from the veterinarian or the pet owner in terms of evaluation periods of the trial.

The study design did not include dogs that received a comparator NSAID molecule to which dogs treated with cimicoxib could be compared and there was no control group that did not receive analgesic treatment. Ethically it is difficult to justify including a negative control group in dogs presented to a veterinarian with pain associated with OA, due to the requirement for analgesia, particularly when data are collected in a field setting with 2 weeks between assessment time points. Including a group that received a different licensed NSAID would have allowed comparison of clinical effect between cimicoxib and an older NSAID molecule, although it is unlikely that differences in analgesic efficacy would have been detected between the two groups of dogs [25] [26]. Furthermore, the relative efficacy of cimicoxib compared with comparator NSAIDs has already been established [6]. However in the survey reported here, where treatment allocation was not randomized, the veterinarian may either subconsciously or consciously selected dogs to receive cimicoxib that they considered would do particularly well on NSAID therapy. It may also have led to selective exclusion of animals based on prognostic factors, for example, dogs with mild disease and pain for which it may have been difficult to discern improvements with NSAID administration [27]. 
Another limitation was the lack of blinding in the study, inherent in a study design where all dogs receive the same drug treatment and this is known by the people carrying out the assessments (veterinarians and dog owners). This knowledge will have subconsciously or otherwise affected outcome measurements, particularly because of the prior expectation that all NSAIDs are analgesic and therefore improvements in lameness and other clinical scoring parameters were expected over the course of treatment [28]. Introduction of a placebo control group would have allowed effects caused by the active treatment (cimicoxib) to be distinguished from favorable effects due to reasons not related to the active treatment, for example a psychological effect on the dog owner or veterinarian. Placebo effects, in both veterinarians and dog owners have been reported in other veterinary studies in dogs and cats where an analgesic treatment was administered for chronic pain associated with OA [29]-[31]. There are a number of other published field studies of clinical efficacy of different NSAIDs for management of OA in dogs that suffer from similar limitations [10] [32] [33].

Serum biochemistry was available for a subset of the dogs that completed the study in order to monitor changes in urea, creatinine, ALT and AST over the 30-day treatment period although collection of blood samples was not a prerequisite for inclusion in the study. Thus, the aim was to collect population data about changes in these biochemical parameters over a 30-day treatment course with cimicoxib, rather than track changes in these variables over time in individual animals. The blood samples were not analysed in a standard manner, with a variety of different analysers being used to measure all biochemical parameters, although studies generally report good consistency between laboratories for the biochemical parameters measured in the present study, and between laboratory and bench top point of care analysers [34]. However knowing this limitation the biochemical data are presented as a distribution compared to a reference value to highlight if there was a change in the whole population distribution and to see if there was a trend towards an increase in "above the reference" group after treatment with cimicoxib independent of the analyser, and the biochemical data were not subject to statistical analyses. The cut off values for each of the parameters that defined a dog to be abnormal or normal are similar to reference values for normality used by many laboratories and were calculated following a synthesis of references values published in the literature. There was a numerical increase in the proportion of dogs with an abnormal serum urea concentration documented at D30 compared with D0, but this was not accompanied by a numerical increase in the proportion of dogs with elevated serum creatinine at D30 compared with D0. Serum urea concentration is influenced by hydration status of the dog and recent dietary protein intake [35], therefore the contribution of cimicoxib administration to the changes in urea is unknown. Compared with changes in serum urea concentration, increased serum creatinine concentration is generally considered to be more specific for altered renal function [36]. The proportion of dogs with abnormal ALT and AST was not numerically different at the start and end of the treatment period, which is in accordance with data reported in other clinical studies monitoring changes in biochemical parameters related to liver damage following NSAID therapy over a similar time course [3].

Gastro-intestinal side effects associated with NSAID administration are a significant cause for concern amongst veterinarians [37], although the prevalence associated with administration of NSAIDs to dogs for OA is difficult to quantify in general practice. Only limited data were collected from owners to probe the occurrence of gastrointestinal side effects associated with cimicoxib administration, nonetheless the majority of owners agreed with the statement that their dog had no visible side effects during the treatment. This suggests that the gastrointestinal side effect profile of cimicoxib is similar to other currently licensed NSAIDs.

\section{Conclusion}

This survey demonstrated improvements in locomotion, mobility, pain scores and dog demeanor and body condition in dogs treated with cimicoxib for thirty days by both owners and veterinarians and provides evidence that these outcome measures, as measured by veterinarians continue to improve after 15 days of treatment up to the 30-day time point. However, these data must be interpreted with knowledge of limitations in the study design, which will have likely resulted in measurement bias for the different outcome variables. Data relating to the use of a new NSAID in the field environment are useful for veterinarians making decisions about which NSAID to administer to dogs that require pain management for OA.

\section{Acknowledgements}

The authors would like to thank Gary Coxon MRCVS for his technical expertise in the design of the study. 


\section{References}

[1] Johnston, S.A. and Budsberg, S.C. (1997) Nonsteroidal Anti-Inflammatory Drugs and Corticosteroids for the Management of Canine Osteoarthritis. Veterinary Clinics of North America: Small Animal Practice, 27, 841-862.

[2] Marshall, W., Bockstahle, B., Hulse, D. and Carmicheal, S. (2009) A Review of Osteoarthritis and Obesity: Current Understanding of the Relationship and Benefit of Obesity Treatment and Prevention in the Dog. Veterinary and Comparative Orthopaedics and Traumatology, 22, 339-345.

[3] Kukanich, B., Bidgood, T. and Knesl, O. (2012) Clinical Pharmacology of Nonsteroidal Anti-Inflammatory Drugs in Dogs. Veterinary Anaesthesia and Analgesia, 39, 69-90. http://dx.doi.org/10.1111/j.1467-2995.2011.00675.x

[4] Henrotin, Y., Sanchez, C. and Balligand, M. (2005) Pharmaceutical and Nutraceutical Management of Canine Osteoarthritis: Present and Future Perspectives. The Veterinary Journal, 170, 113-123. http://dx.doi.org/10.1016/j.tvjl.2004.08.014

[5] Hercock, C.A., Pinchbeck, G., Giejda, A., Clegg, P.D. and Innes, J.F. (2009) Validation of a Client-Based Clinical Metrology Instrument for the Evaluation of Canine Elbow Osteoarthritis. Journal of Small Animal Practice, 50, 266-271. http://dx.doi.org/10.1111/j.1748-5827.2009.00765.x

[6] EMA EPAR 2011 Cimalgex. European Public Assessment Report. London.

[7] (2012) IRIS Staging of CKD. http://www.iris-kidney.com/guidelines/en/staging_ckd.shtml

[8] Autefage, A., Palissier, F.M., Asimus, E. and Pepin-Richard, C. (2011) Long-Term Efficacy and Safety of Firocoxib in the Treatment of Dogs with Osteoarthritis. Veterinary Record, 168, 617-621. http://dx.doi.org/10.1136/vr.d1456

[9] Bath, P.M., Macleod, M.R. and Green, A.R. (2009) Emulating Multicentre Clinical Stroke Trials: A New Paradigm for Studying Novel Interventions in Experimental Models of Stroke. International Journal of Stroke, 4, 471-479. http://dx.doi.org/10.1111/j.1747-4949.2009.00386.x

[10] Mansa, S., Palmér, E., Grøndahl, C., Lønaas, L. and Nyman, G. (2007) Long-Term Treatment with Carprofen of 805 Dogs with Osteoarthritis. Veterinary Record, 160, 427-430. http://dx.doi.org/10.1136/vr.160.13.427

[11] Edamura, K., King, J.N., Seewald, W., Sakakibara, N. and Okumura, M. (2012) Comparison of Oral Robenacoxib and Carprofen for the Treatment of Osteoarthritis in Dogs: A Randomized Clinical Trial. The Journal of Veterinary Medical Science, 74, 1121-1131. http://dx.doi.org/10.1292/jvms.11-0529

[12] Holton, L.L., Scott, E.M., Nolan, A.M., Welsh, E. and Flaherty, D. (1998) Comparison of Three Methods Used for Assessment of Pain in Dogs. Journal of the American Veterinary Medical Association, 212, 61-66.

[13] Hielm-Björkman, A.K., Kapatkin, A.S. and Rita, H.J. (2011) Reliability and Validity of a Visual Analogue Scale Used by Owners to Measure Chronic Pain Attributable to Osteoarthritis in Their Dogs. American Journal of Veterinary Research, 72, 601-607. http://dx.doi.org/10.2460/ajvr.72.5.601

[14] Hielm-björkman, A.K., Kuusela, E., Liman, A., Markkola, A., Saarto, E., Huttunen, P., Leppäluoto, J., Tulamo, R.M. and Raekalio, M. (2003) Evaluation of Methods for Assessment of Pain Associated with Chronic Osteoarthritis in Dogs. Journal of the American Veterinary Medical Association, 222, 1552-1558. http://dx.doi.org/10.2460/javma.2003.222.1552

[15] Brown, D.C., Boston, R., Coyne, J.C. and Farrar, J.T. (2008) Ability of the Canine Brief Pain Inventory to Detect Response to Treatment in Dogs with Osteoarthritis. Journal of the American Veterinary Medical Association, 233, 12781283. http://dx.doi.org/10.2460/javma.233.8.1278

[16] Hudson, J.T., Slater, M.R., Taylor, L., Scott, H.M. and Kerwin, S.C. (2004) Assessing Repeatability and Validity of a Visual Analogue Scale Questionnaire for Use in Assessing Pain and Lameness in Dogs. American Journal of Veterinary Research, 65, 1634-1643. http://dx.doi.org/10.2460/ajvr.2004.65.1634

[17] Wiseman-Orr, M.L., Scott, E.M., Reid, J. and Nolan, A.M. (2006) Validation of a Structured Questionnaire as an Instrument to Measure Chronic Pain in Dogs on the Basis of Effects on Health-Related Quality of Life. American Journal of Veterinary Research, 67, 1826-1836. http://dx.doi.org/10.2460/ajvr.67.11.1826

[18] Rao, P. and Knaus, E.E. (2008) Evolution of Nonsteroidal Anti-Inflammatory Drugs (NSAIDs): Cyclooxygenase (COX) Inhibition and Beyond. Journal of Pharmacy \& Pharmaceutical Sciences, 11, 81s-110s.

[19] Burian, M. and Geisslinger, G. (2005) Cox-Dependent Mechanisms Involved in the Antinociceptive Action of NSAIDs at Central and Peripheral Sites. Pharmacology \& Therapeutics, 107, 139-154. http://dx.doi.org/10.1016/j.pharmthera.2005.02.004

[20] Innes, J.F., Clayton, J. and Lascelles, B.D.X. (2010) Review of the Safety and Efficacy of Long-Term NSAID Use in the Treatment of Canine Osteoarthritis. Veterinary Record, 166, 226-230. http://dx.doi.org/10.1136/vr.c97

[21] Strand, V., Simon, L.S., Dougados, M., Sands, G.H., Bhadra, P., Breanza, A. and Immitt, J. (2011) Treatment of Osteoarthritis with Continuous versus Intermittent Celecoxib. Journal of Rheumatology, 38, 2625-2634.

http://dx.doi.org/10.3899/jrheum.110636 
[22] Wiseman-Orr, M.L., Nolan, A.M., Reid, J. and Scott, E.M. (2004) Development of a Questionnaire to Measure the Effects of Chronic Pain on Health-Related Quality of Life in Dogs. American Journal of Veterinary Research, 65, 10771084. http://dx.doi.org/10.2460/ajvr.2004.65.1077

[23] Hielm-björkman, A.K., Rita, H. and Tulamo, R.M. (2009) Psychometric Testing of the Helsinki Chronic Pain Index by Completion of a Questionnaire in Finnish by Owners of Dogs with Chronic Signs of Pain Caused by Osteoarthritis. American Journal of Veterinary Research, 70, 727-734. http://dx.doi.org/10.2460/ajvr.70.6.727

[24] Innes, J.F. and Barr, A.R. (1998) Can Owners Assess Outcome Following Treatment of Canine Cruciate Ligament Deficiency? Journal of Small Animal Practice, 39, 373-378. http://dx.doi.org/10.1111/j.1748-5827.1998.tb03735.x

[25] Papich, M.G. (2008) An Update on Nonsteroidal Anti-Inflammatory Drugs (NSAIDs) in Small Animals. Veterinary Clinics of North America: Small Animal Practice, 38, 1243-1266. http://dx.doi.org/10.1016/j.cvsm.2008.09.002

[26] Reymond, N., Speranza, C., Gruet, P., Seewald, W. and King, J.N. (2011) Robenacoxib vs. Carprofen for the Treatment of Canine Osteoarthritis; A Randomized, Noninferiority Clinical Trial. Journal of Veterinary Pharmacology and Therapeutics, 35, 175-183. http://dx.doi.org/10.1111/j.1365-2885.2011.01297.x

[27] Vesterinen, H.V., Egan, K., Deister, A., Schlattmann, P., Macleod, M.R. and Dirnagl, U. (2011) Systematic Survey of the Design, Statistical Analysis, and Reporting of Studies Published in the 2008 Volume of the Journal of Cerebral Blood Flow and Metabolism. Journal of Cerebral Blood Flow \& Metabolism, 31, 1064-1072. http://dx.doi.org/10.1038/jcbfm.2010.217

[28] Dirnagl, U. and Macleod, M.R. (2009) Stroke Research at a Road Block: The Streets from Adversity Should Be Paved with Meta-Analysis and Good Laboratory Practice. British Journal of Pharmacology, 157, 1154-1156. http://dx.doi.org/10.1111/j.1476-5381.2009.00211.x

[29] Lascelles, B.D., Hansen, B.D., Roe, S., Depuy, V., Thomson, A., Pierce, C.C., Smith, E.S. and Rowinski, E. (2007) Evaluation of Client-Specific Outcome Measures and Activity Monitoring to Measure Pain Relief in Cats with Osteoarthritis. Journal of Veterinary Internal Medicine, 21, 410-416. http://dx.doi.org/10.1111/j.1939-1676.2007.tb02983.X

[30] Malek, S., Sample, S.J., Schwartz, Z., Nemke, B., Jacobson, P.B., Cozzi, E.M., Schaefer, S.L., Bleedorn, J.A., Holzman, G. and Muir, P. (2012) Effect of Analgesic Therapy on Clinical Outcome Measures in a Randomized Controlled Trial Using Client-Owned Dogs with Hip Osteoarthritis. BMC Veterinary Research, 8, 185. http://dx.doi.org/10.1186/1746-6148-8-185

[31] Conzemius, M.G. and Evans, R.B. (2012) Caregiver Placebo Effect for Dogs with Lameness from Osteoarthritis. Journal of the American Veterinary Medical Association, 241, 1314-1319. http://dx.doi.org/10.2460/javma.241.10.1314

[32] Budsberg, S.C., Johnston, S.A., Schwarz, P.D., Decamp, C.E. and Claxton, R. (1999) Efficacy of Etodolac for the Treatment of Osteoarthritis of the Hip Joints in Dogs. Journal of the American Veterinary Medical Association, 214, 206-210.

[33] Ryan, W.G., Moldave, K. and Carithers, D. (2007) Clinical Effectiveness and Safety of a New NSAID, Firocoxib: A 1,000 Dog Study. Veterinary Therapeutics, 7, 119-126.

[34] Concordet, D., Vergez, F., Trumel, C., Diquélou, A., lanore, D., Le Garrérès, A., Pagès, J.P., Péchereau, D., Médaille, C. and Braun, J.P. (2008) A Multicentric Retrospective Study of Serum/Plasma Urea and Creatinine Concentrations in Dogs Using Univariate and Multivariate Decision Rules to Evaluate Diagnostic Efficiency. Veterinary Clinical Pathology, 37, 96-103. http://dx.doi.org/10.1111/j.1939-165X.2008.00007.x

[35] Ulleberg, T., Robben, J., Nordahl, K.M., Ulleberg, T. and Heiene, R. (2011) Plasma Creatinine in Dogs: Intra- and InterLaboratory Variation in 10 European Veterinary Laboratories. Acta Veterinaria Scandinavica, 53, 25. http://dx.doi.org/10.1186/1751-0147-53-25

[36] Hosten, A.O. (1990) BUN and Creatinine in Clinical Methods. In: Walker, H.K., Hall, W.D. and Hurst, J.W., Eds., The History, Physical, and Laboratory Examinations, 3rd Edition, Butterworths, Boston, 874-878.

[37] Williams, V.M., Lascelles, B.D. and Robson, M.C. (2005) Current Attitudes to, and Use of, Peri-Operative Analgesia in Dogs and Cats by Veterinarians in New Zealand. New Zealand Veterinary Journal, 53, 193-202.

http://dx.doi.org/10.1080/00480169.2005.36504 